\title{
Individual Differences in Anterior Cingulate Activation Associated with Attentional Bias Predict Cocaine Use After Treatment
}

\author{
Reshmi Marhe', Maartje Luijten ${ }^{1,2}$, Ben JM van de Wetering ${ }^{3}$, Marion Smits ${ }^{2}$ and Ingmar HA Franken*,1,4 \\ 'Department of Psychology, Erasmus University Rotterdam, Rotterdam, The Netherlands; '2 Department of Radiology, Erasmus MC - University \\ Medical Center Rotterdam, Rotterdam, The Netherlands; ${ }^{3}$ Bouman-GGZ, Rotterdam, The Netherlands; ${ }^{4}$ Department of Child and Adolescent \\ Psychiatry, Erasmus MC - University Medical Center Rotterdam, Rotterdam, The Netherlands
}

\begin{abstract}
Drug-dependent patients often relapse into drug use after treatment. Behavioral studies show that enhanced attentional bias to drug cues is a precursor of relapse. The present functional magnetic resonance imaging ( $\mathrm{fMRI}$ ) study examined whether brain regions involved in attentional bias are predictive of cocaine use after treatment. Attentional bias-related brain activity was measured — with a cocaine Stroop task - in cocaine-dependent patients during their first week in detoxification treatment and was used to predict cocaine use at 3-month follow-up. The predictive value of attentional bias-related brain activity in a priori defined regions of interest, in addition to other measures such as self-reports of substance severity, craving, and behavioral attentional bias were examined. The results show that craving in the week before treatment and individual variability in attentional bias-related activity in the dorsal anterior cingulate cortex (dACC) were significant predictors of days of cocaine use at 3-month follow-up and accounted for $45 \%$ in explained variance. Brain activity in the dACC uniquely contributed $22 \%$ of explained variance to the prediction model. These findings suggest that hyperactive attentional biasrelated brain activity in the AACC might be a biomarker of relapse vulnerability as early as in the first week of detoxification treatment. Ultimately, this may help to develop individually tailored treatment interventions to reduce relapse risk.

Neuropsychopharmacology (2013) 38, 1085-1093; doi:I0.1038/npp.2013.7; published online 30 January 2013
\end{abstract}

Keywords: cocaine; relapse; dorsal anterior cingulate cortex; attentional bias; functional MRI; addiction Stroop task

\section{INTRODUCTION}

The main goal of most substance dependence treatment is to prevent patients from relapsing into substance use. Despite the efforts to treat substance-dependent patients effectively, treatment dropout rates are generally $>50 \%$ and most patients subsequently relapse (Miller, 1996; Franken and Hendriks, 1999; Hättenschwiler et al, 2000). The identification of predictors of substance use relapse have been of high interest in addiction research (eg, Miller et al, 1996; Donovan, 1996; McKay, 1999). Particularly in cocaine addiction, a variety of predictors of relapse have been tested including demographic variables, substance use severity, and craving (for a review, see Poling et al, 2007). These predictors are largely based on self-report measures. However, over the years the idea that addiction is a relapsing brain disorder (Leshner, 1997) has gained more interest. Support for this idea comes from recent studies

\footnotetext{
*Correspondence: Dr HA Franken, Institute of Psychology, Erasmus University Rotterdam, P.O. Box 1738, Rotterdam 3000 DR, The Netherlands, Tel: +31 10408 9563, Fax: +31 104089009 , E-mail: franken@fsw.eur.nl

Received 21 August 2012; revised 18 December 2012; accepted 20 December 2012; accepted article preview online 7 January 2013
}

suggesting that (neuro)cognitive measures might be better predictors of relapse than self-report measures (Marissen et al, 2006; Kosten et al, 2006). Currently, addiction research is focusing more on the role of cognitive predictors in relapse to substance use, and specifically the neural correlates of cognitive control (Garavan and Hester, 2007; Goldstein and Volkow, 2011).

Attentional bias is one of the most studied cognitive processes in addiction. Attentional bias in addiction refers to the automatically enhanced cognitive processing of drugrelated (salient) stimuli compared with neutral (nonsalient) stimuli. Recent theories suggest that it is associated with craving and therefore has an important role in the maintenance and relapse of drug dependency (Franken, 2003; Field and Cox, 2008). A widely used task to measure attentional bias is the addiction Stroop task (Cox et al, 2006). Behavioral studies that have used the addiction Stroop paradigm in substance-dependent individuals have indeed found an attentional bias toward the substance of abuse (for a review, see Cox et al, 2006). More importantly, several studies show that attentional bias to substancerelated stimuli (on the behavioral level) is a predictor of treatment outcome and relapse in smoking (Waters et al, 2003; Powell et al, 2010), alcohol (Cox et al, 2002, 2007), 
heroin (Marissen et al, 2006), and cocaine use (Carpenter et al, 2006; Marhe et al, 2012). These results indicate a possible clinical relevance of attentional bias. In addition, more recent studies have taken an interest in studying the neural substrates of attentional bias in addiction.

Brain regions that have been found to be associated with attentional bias in addiction include (prefrontal) cortices such as the anterior cingulate cortex (ACC; Luijten et al, 2011, 2012; Nestor et al, 2011; Vollstädt-Klein et al, 2012), dorsolateral prefrontal cortex (DLPFC; Hester and Garavan, 2009; Luijten et al, 2012; Vollstädt-Klein et al, 2012), insula (Luijten et al, 2011; Vollstädt-Klein et al, 2012), and subcortical structures such as the nucleus accumbens (Nestor et al, 2011) and amygdala (Vollstädt-Klein et al, 2012; Janes et al, 2010a). Most pertinent to the present study is that attentional bias-related brain activity in the insula and dorsal ACC (dACC) has been found to be predictive of smoking relapse (Janes et al, 2010b). Involvement of the ACC in response to cocaine cues has also been found in a study with cocaine users (using the addiction Stroop task; Goldstein et al, 2009). However, no study to date has examined whether brain regions involved in attentional bias to cocaine stimuli (as measured by a Stroop task) are predictive of cocaine relapse after treatment.

The goal of the present study therefore was to examine whether brain activity during a cocaine Stroop task measured in the first week of detoxification treatment would be associated with cocaine use measured at 3-month follow-up. Based on aforementioned studies, we hypothesized that brain regions involved in attentional bias (ie, dACC, DLPFC, insula, nucleus accumbens, and amygdala) would be associated with cocaine use at follow-up.

\section{MATERIALS AND METHODS}

\section{Participants}

Participants were 34 cocaine-dependent inpatients recruited from an addiction treatment center (Bouman-GGZ) in Rotterdam, the Netherlands. Inclusion criteria were (1) age between 18 and 65 years; (2) DSM-IV diagnosis for cocaine dependence (assessed by both a physician and a research psychologist); and (3) the ability to speak, read, and write in Dutch at an eighth-grade literacy level. Exclusion criteria were (1) indications of severe psychopathology (ie, psychosis and severe mood disorder, as assessed by a physician); (2) self-reported color blindness or (noncorrected) defective vision; (3) pregnancy or breast-feeding; and (4) functional magnetic resonance imaging (fMRI) contra-indications. We had to exclude the data of eight participants. Reasons for exclusion were loss to follow-up $(n=1)$; mismatch between self-reported days of cocaine use and urine results at follow-up $(n=1)$; vision problems $(n=2)$; an accuracy rate on the Stroop task of $<60 \%(n=3$; these participants also showed excessive head motion of $>3 \mathrm{~mm}$ ); major anatomical abnormality observed on MRI $(n=1)$. The final sample consisted of 26 cocaine-dependent patients (for demographics, see Table 1).

Approval of the study was received from the Ethics Committee of the Erasmus MC-University Medical Center Rotterdam, Rotterdam, the Netherlands. All procedures were carried out with the adequate understanding and written informed consent of the participants. All participants received a financial compensation of 35 euros after completion of the fMRI measure and 25 euros after completion of the 3-month follow-up measure. The present study was part of a larger study that investigated relapse using other psychophysiological and cognitive measures (not reported in the present study).

\section{Stroop Task}

A cocaine Stroop task was used to measure attentional bias for cocaine-related stimuli. The task contained 10 cocaine words (Dutch equivalent of basepipe, crack, smoke, cocaine, blow, line, coke, snort, powder, and base), 10 neutral words drawn from the category 'indoor features' (Dutch equivalent of blanket, sofa, oven, lamp, attic, cabinet, armchair, tap, couch, and stove), and 10 letter strings (eg, MMMM; Waters et al, 2012). Letter strings were included to increase the number of conditions in the task which, in combination with a semi-random block order, reduced multicollinearity between parameter estimates for cocaine and neutral words (see Supplementary Information, Part I for more information on the order of task conditions). Words and letter strings were presented in the colors blue, yellow, green, or red. Participants were instructed that they should ignore the meaning of the word and only respond to the color that the word was presented in. They were instructed to indicate as rapidly and accurately as possible in which color the word was presented by pressing one of four response buttons with corresponding colors.

Table I Demographic and Substance Use Variables ${ }^{a}$

\begin{tabular}{|c|c|}
\hline Subject variable & $\begin{array}{l}\text { Final sample } \\
\quad(n=26)\end{array}$ \\
\hline \multicolumn{2}{|l|}{ Demographic variables } \\
\hline Age & $38.7(9.2)$ \\
\hline Males (\%) & 85 \\
\hline \multicolumn{2}{|l|}{ Education (\%) } \\
\hline Primary education & 4 \\
\hline Junior secondary education & 54 \\
\hline Senior secondary education & 31 \\
\hline Higher education & 11 \\
\hline \multicolumn{2}{|l|}{ Substance use variables } \\
\hline Total years of cocaine use & $11.4(6.3)$ \\
\hline $\begin{array}{l}\text { Number of days of cocaine use in } 30 \text { days before } \\
\text { treatment entry }\end{array}$ & | $6.5(\mid 1.9)$ \\
\hline \multicolumn{2}{|l|}{ Cocaine administration route (\%) } \\
\hline Snorting & 31 \\
\hline Smoking & 65 \\
\hline Intravenous & 4 \\
\hline \multicolumn{2}{|l|}{ Craving in the week before treatment } \\
\hline OCDUS desire and control $(\mid-5)$ & $2.8(1.1)$ \\
\hline
\end{tabular}

Abbreviation: OCDUS, Obsessive Compulsive Drug Use Scale.

${ }^{a} V$ alues are means (SD) unless otherwise indicated (\%). 
The task started with 32 practice trials consisting of only letter strings. Next, in the experimental phase, stimuli were presented in a blocked design with three categories (cocaine, neutral, and letter strings). Every category was presented six times, resulting in a total of 18 blocks. Each block contained 10 words. After each six blocks, there was a 38-s resting period. Order of presentation of the task conditions was semi-random; block order was the same for each participant. Each experimental trial began with a 250-ms fixation cross, followed by stimulus presentation with a duration of $1750 \mathrm{~ms}$. Response could be given within this timeframe. After response, the stimulus remained on screen. No feedback regarding performance accuracy was provided to the participants.

\section{Procedure}

Patients entered the treatment center for an inpatient detoxification treatment. A standard detoxification treatment in this setting has a duration of 3 weeks. The specific goal of this treatment is to reduce cocaine withdrawal symptoms by means of psychoeducation about the detox symptoms and individual therapy based on cognitivebehavioral techniques. Afterwards, the patients start a follow-up treatment at a different department within the same treatment center. This is usually a rehabilitation program with a variable duration between 1 month and 2 years, depending on the need for treatment.

Patients that met the study criteria were informed about the study on the second day of their detoxification treatment. They had $24 \mathrm{~h}$ to decide whether to participate. Volunteers signed the informed consent on the third day of detoxification treatment. Cocaine use severity was assessed using the Addiction Severity Index (ASI; McLellan et al, 1980; Hendriks et al, 1989) and past week craving using the cocaine version of the Obsessive Compulsive Drug Use Scale (OCDUS; Franken et al, 2002). On the fourth day of treatment, participants were escorted to the Erasmus Medical Center where they performed the cocaine Stroop task in the scanner. After completion, participants were escorted back to the treatment center.

To ensure retention and compliance for the follow-up assessment, the researcher first collected multiple contact information at the start of the study. For example, we collected phone numbers and e-mail addresses of the participant but also of their family, friends, social workers, and other professionals involved with the patient. Second, the participant was informed that (s)he would receive a financial compensation of 25 euros for completion of the follow-up assessment. Third, participants were ensured that information on current use (both self-reports and urine screens) was used for research purposes only. Participants were contacted 3 months after study participation via telephone and/or e-mail to set up an appointment for a faceto-face follow-up interview. Follow-up tests were performed in the treatment center. If a participant was out of treatment at this point, then he/she was asked to return once to the treatment center to complete the follow-up tests. If the participant was unable to travel to the main treatment center for the assessment, then the researcher would perform the assessment in the (for the participant) nearest Bouman-GGZ treatment facility. If that was also not possible, then we arranged transfer by taxi for the participant to the treatment center.

\section{Outcome Measure}

The number of days of recent cocaine use was measured 3 months after study participation. At follow-up, participants were asked to report the number of days they had used cocaine in the last 30 days, which we labeled 'recent cocaine use'. Self-reports were biochemically verified by means of urine screens. All self-reports of 0 days of use in the last 30 days were confirmed by a negative urine screen, except for one participant who reported 0 days of use while the urine screen was positive. This participant was excluded from the analysis (see Participants section). In the final sample, 11 participants reported not to have used cocaine in the last month and 15 participants reported to have used cocaine 12.1 days on average (range 1-30, SD 10.6) in the last month.

\section{fMRI Data Acquisition and Processing}

Imaging data were obtained with a 3T GE Healthcare MRI scanner. Blood oxygen level-dependent (BOLD) sensitive functional echo-planar imaging $\mathrm{T} 2^{*}$-weighted images were acquired in 34 axial slices (thickness $=2.6 \mathrm{~mm}$, interslice gap $=0.4 \mathrm{~mm}$ ) covering the entire supratentorial brain with a repetition time (TR) of $2500 \mathrm{~ms}$, echo time (TE) of $30 \mathrm{~ms}$, field of view (FOV) of $220 \mathrm{~mm}$, and matrix size of $64 \times 64$. A structural 3-dimensional inversion recovery (IR) fast spoiled gradient recalled echo (FSPGR) T1-weighted image was acquired in 192 axial slices (thickness $=1.6$ and $0.8 \mathrm{~mm}$ overlap, resulting in an effective slice thickness of $0.8 \mathrm{~mm}$ ) with TR of $12.0 \mathrm{~ms}$, TE of $3.7 \mathrm{~ms}$, a rectangular FOV of $250 \times 175 \mathrm{~mm}^{2}$, and matrix size of $416 \times 256 \mathrm{~mm}$. Due to an unexpected scanner shutdown, we were forced to continue the project on a different 3T scanner by the same vendor (GE Healthcare, Milwaukee, WI, USA) during the study. Data of $n=8$ were acquired on the Signa HDxt scanner and data of $n=18$ were acquired on the Discovery MR750 scanner. In Supplementary Information (Part VII), we describe additional analyses regarding the use of two scanners. There we report temporal signal-to-noise maps of both scanners and we conducted the main statistical analyses with scanner type included as covariate, showing that it is unlikely that the use of two scanners influenced the present findings.

Functional images were analyzed using SPM8 (Statistical Parametric Mapping; Wellcome Trust Centre for Neuroimaging, London, UK). Preprocessing of the functional data included realignment and unwarping of all functional images. Next, the anatomical scan was co-registered to the mean $\mathrm{T}^{*}$-weighted image and subsequently segmented into gray and white matter. Segmentation parameters were used for normalization using the SPM T1 MNI 512 template. Normalized images were spatially smoothed with an 8-mm full-width at half-maximum Gaussian filter. The three task conditions, cocaine words, neutral words, and letters were modeled in the context of the general linear model, using delta functions convolved with a canonical hemodynamic response function (error rates were not associated with activation estimates, see Supplementary Information, 
Part IV for results). The contrast reflecting brain activation associated with attentional bias (cocaine words minus neutral words) was calculated for each individual. Subsequently, five a priori regions of interest (ROIs) were selected based on their presumed role in attentional bias, including the bilateral dACC, DLPFC, insula, nucleus accumbens, and amygdala (Figure 1; see Introduction section for rationale). ROIs for the DLPFC, insula, and amygdala were derived from the automatic anatomical labeling (AAL) atlas (Tzourio-Mazoyer et al, 2002). Since the dACC and nucleus accumbens are not included in the AAL atlas, an $8-\mathrm{mm}$ sphere around MNI coordinates \pm 83032 was used as an ROI for the dACC (Mars et al, 2005) and an 8-mm sphere around MNI coordinates $\pm 10 \quad 12-2$ was used for the nucleus accumbens (Knutson et al, 2008). To test the main attentional bias effect in the ROIs, the cocaine minus neutral words contrast was entered in a random effects one-sample $t$-test. Results were thresholded at $p<0.05$, Family Wise Error (FWE) corrected for multiple comparisons across the search volume (small volume correction: Friston et al, 1996; Worsley et al, 1996). To do so, analyses were first thresholded at $p<0.001$ uncorrected with 20 contingently activated voxels $\left(160 \mathrm{~mm}^{3}\right.$; see Supplementary Information, Part V), and then corrected using a small volume correction $(p<0.05$ FWE corrected) in which the search volume was restricted by the a priori defined ROIs.

\section{Statistical Analyses}

First, to demonstrate attentional bias on a behavioral level for cocaine words, behavioral data of the Stroop task were examined (means are displayed in Table 2). Differences in reaction times (RTs) and accuracy scores for the cocaine words and the neutral words were tested using a paired samples $t$-test. To use the Stroop effect as a predictor in regression models (described below), a single differential score was calculated for RT and for accuracy (by subtracting results on cocaine words from neutral words).

Second, linear regression analysis was used to examine the predictive value of multiple variables (ie, addiction severity, craving, behavioral and brain-related attentional bias) in the prediction of the outcome measure 'recent cocaine use'. Due to the relative large amount of predictor variables, a stepwise regression analysis was performed (Field, 2009). The stepwise method tests, at each addition of a variable into the regression model, which variable contributes the least to the prediction of recent cocaine use and removes this variable from the model. By using this method all redundant predictors are filtered out of the model. Because we were interested in the unique contribution of three clusters of variables (ie, self-reports, behavior, and imaging) we performed the regression analysis in three steps. Step 1 contained four self-report variables: total years of cocaine use, number of days of cocaine use in 30 days before treatment entry, and cocaine administration route (ie, addiction severity) as well as craving in the week before treatment. Since cocaine administration route is a categorical variable with three levels, two dummy variables were created. Step 2 contained two variables of behavioral data: Stroop effect RT and Stroop effect accuracy. Step 3 contained the imaging variables (extracted contrast values for cocaine minus neutral words in the five bilateral ROIs; left and right dACC, left and right DLPFC, left and right insula, left and right nucleus accumbens, left and right amygdala). In each step, the selection criteria for inclusion and exclusion of predictors in the model were $F_{\text {enter }}: p \leqslant 0.05$ and $F_{\text {remove }}: p \geqslant 0.10$.

Third, we wanted to check the robustness of the individual predictors that were included in the model based on the results of the stepwise regression. To do so, bootstrapping was used with 2000 bootstrapped samples and a 95\% confidence interval (CI; Efron and Tibshirani, 1993; Mooney and Duval, 1993). The bootstrap procedure

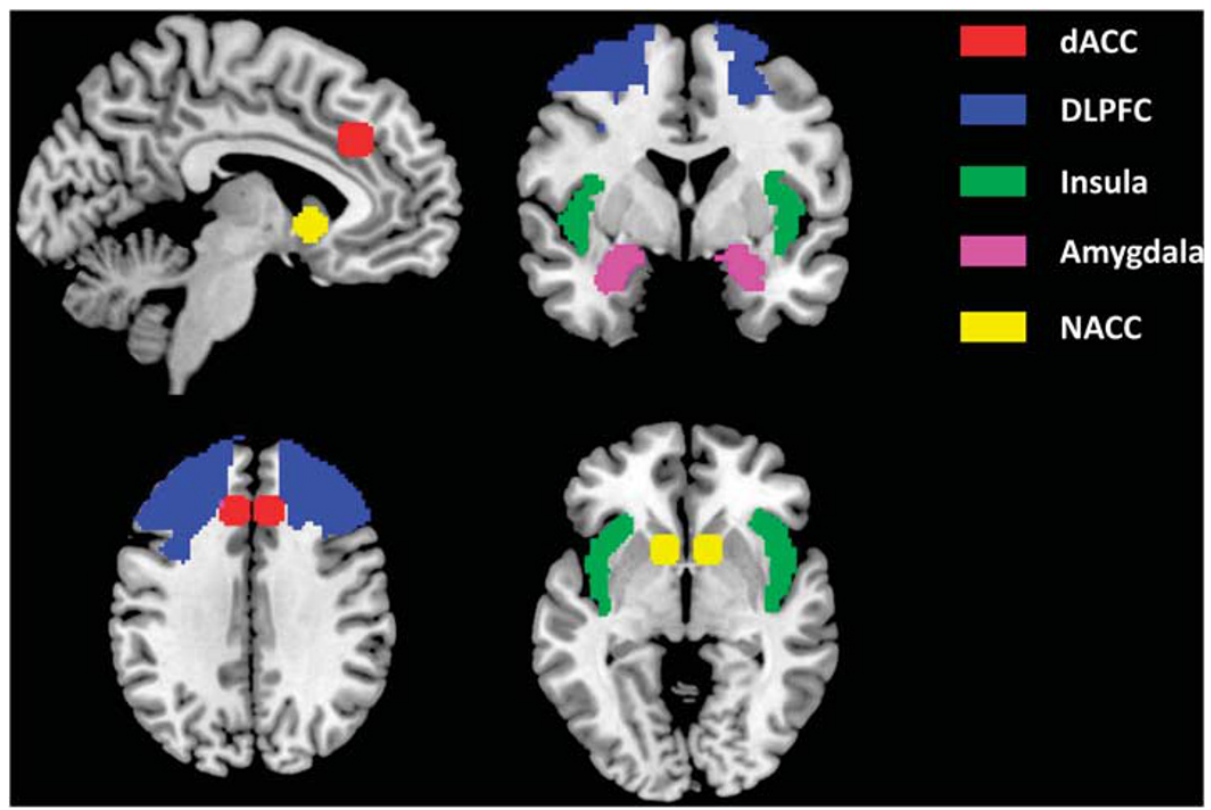

Figure I A priori defined, literature-based regions of interest involved in attentional bias to substance cues. dACC, dorsal anterior cingulate cortex; DLPFC, dorsolateral prefrontal cortex; NACC, nucleus accumbens. 
can only be conducted when predictor variables are forcedly entered into the regression model (instead of stepwise). Therefore, predictors that met the inclusion criteria of the stepwise method were entered in a hierarchical regression analysis.

Finally, predictors were checked for multicollinearity by means of tolerance statistics. All statistical analysis were conducted with SPSS 18.0 (IBM Corporation, Armonk, NY, USA).

\section{RESULTS}

\section{Attentional Bias}

We found a significant difference between Stroop RTs on cocaine words and neutral words, $(t[25]=6.87, p<0.001)$, with cocaine-dependent patients responding slower to cocaine-related stimuli than to neutral stimuli. This indicates the presence of an attentional bias. No differences in accuracy between cocaine and neutral words were found, $(t[25]=-1.84, n s)$.

For the imaging data, we did not find significant activation $(p<0.05$ FWE small volume corrected) for the cocaine minus neutral words contrast in any of the ROIs within the patient sample.

\section{Predictors of Recent Cocaine Use}

The stepwise regression yielded that two predictorscraving in the week before treatment and attentional biasrelated activity in the right dACC-met the selection criterion for inclusion in the model. Other self-report, behavioral, and imaging variables were excluded based on the criterion (see Table 3a). Model statistics are displayed in Table $3 \mathrm{~b}$. The first step, including craving in the week before treatment, accounted for $23 \%$ of explained variance in recent cocaine use. Individual differences in brain activation for the attentional bias contrast in the right dACC added another 22\% explained variance to the model. Thus, together both predictors accounted for $45 \%$ of explained variance in the prediction of recent cocaine use.

Bootstrapping confirmed that both individual predictors were robust predictors of treatment outcome (see Table 3c). The coefficients indicated a positive association between the predictors and the outcome measure. These findings showed that higher self-reported past-week craving and enhanced activation for cocaine words $v s$ neutral words in the right dACC (both measured during the first week of treatment) were associated with more days of recent cocaine

Table 2 Mean Reaction Times (milliseconds) and Accuracy Scores (percentages) for the Stroop Task

\begin{tabular}{lcr}
\hline & Mean & SD \\
\hline RT cocaine words & 972 & 137 \\
RT neutral words & 886 & 126 \\
Accuracy cocaine words & 91 & 8 \\
Accuracy neutral words & 93 & 8
\end{tabular}

Abbreviation: RT, reaction time. use (measured at 3-month follow-up). Direct associations between each significant predictor and recent cocaine use are displayed in Figure 2.

Finally, collinearity statistics indicated that the predictors were not associated with each other (tolerance $=0.98$ ).

Table 3a Statistics of Predictor Variables Added in the Stepwise Regression Analysis

\begin{tabular}{|c|c|c|c|}
\hline Predictor variables & $\begin{array}{c}\text { Standardized } \\
\text { coefficients }(=\boldsymbol{\beta})\end{array}$ & t statistic & $p$-Value \\
\hline \multicolumn{4}{|l|}{ Self-reports } \\
\hline Total years of cocaine use & 0.14 & 0.88 & 0.387 \\
\hline $\begin{array}{l}\text { Number of days of cocaine use in } \\
30 \text { days before treatment entry }\end{array}$ & 0.04 & 0.22 & 0.831 \\
\hline \multicolumn{4}{|l|}{ Cocaine administration route } \\
\hline Snorting vs smoking & 0.18 & 1.09 & 0.287 \\
\hline Snorting vs intravenous & 0.08 & 0.46 & 0.647 \\
\hline $\begin{array}{l}\text { Craving in the week before } \\
\text { treatment }\end{array}$ & 0.41 & 2.57 & $0.017^{\mathrm{a}}$ \\
\hline \multicolumn{4}{|l|}{ Behavior } \\
\hline Stroop effect RT & -0.24 & $-|.5|$ & 0.145 \\
\hline Stroop effect accuracy & -0.11 & -0.66 & 0.519 \\
\hline \multicolumn{4}{|l|}{ Imaging $^{\mathrm{b}}$} \\
\hline I-dACC & -0.53 & -1.70 & 0.104 \\
\hline$r-d A C C$ & 0.47 & 3.00 & $0.006^{\mathrm{a}}$ \\
\hline I-DLPFC & -0.35 & -1.39 & 0.178 \\
\hline$r$-DLPFC & -0.27 & -1.01 & 0.322 \\
\hline I-insula & -0.36 & -1.41 & 0.174 \\
\hline r-insula & -0.06 & 0.21 & 0.832 \\
\hline I-nucleus accumbens & -0.11 & -0.40 & 0.694 \\
\hline r-nucleus accumbens & -0.05 & -0.17 & 0.868 \\
\hline I-amygdala & -0.37 & -1.53 & 0.140 \\
\hline r-amygdala & 0.04 & 0.15 & 0.884 \\
\hline
\end{tabular}

Abbreviations: RT, reaction time; I, left; r, right; dACC, dorsal anterior cingulate cortex; DLPFC, dorsolateral prefrontal cortex.

${ }^{a}$ Meet selection criteria $F_{\text {enter: }} p \leqslant 0.05$ and $F_{\text {remove: }} p \geqslant 0.10$.

${ }^{\mathrm{b}}$ Attentional bias contrast (cocaine minus neutral).

Table 3b Model Statistics and Change Statistics of the Final Model Predicting Recent Cocaine Use

\begin{tabular}{|c|c|c|c|c|c|c|}
\hline \multirow{2}{*}{$\begin{array}{l}\text { Predictor } \\
\text { variables }\end{array}$} & \multicolumn{3}{|c|}{ Model statistics } & \multicolumn{3}{|c|}{ Change statistics } \\
\hline & $R^{2}$ & $\begin{array}{c}\text { F } \\
\text { Statistic }\end{array}$ & $\begin{array}{c}p- \\
\text { Value }\end{array}$ & $\Delta R^{2}$ & $\underset{\text { statistic }}{\Delta \mathbf{F}}$ & $\begin{array}{c}\Delta p- \\
\text { Value }\end{array}$ \\
\hline Step 1 & 0.23 & 7.15 & 0.013 & & & \\
\hline \multicolumn{7}{|c|}{ Craving in the week before treatment } \\
\hline $\begin{array}{l}\text { Step } 2 \\
\quad r-d A C C^{a}\end{array}$ & 0.45 & 9.26 & 0.001 & 0.22 & 9.00 & 0.006 \\
\hline
\end{tabular}

Abbreviations: $\mathrm{dACC}$, dorsal anterior cingulate cortex; r, right.

${ }^{a}$ Attentional bias contrast (cocaine minus neutral). 


\section{DISCUSSION}

The present study is the first to examine to what extent attentional bias-related brain activity predicts recent cocaine use after treatment, over and above other relevant predictors such as craving, substance use severity, and behavioral measures of attentional bias. We found that both higher levels of self-reported craving in the week before treatment and enhanced attentional bias-related brain activity in the right dACC-measured in the first week of detoxification treatment-were associated with cocaine use at 3-month follow-up. Cocaine use severity, behavioral measures of attentional bias, and other brain regions that have previously been shown to be associated with attentional bias (ie, DLPFC, insula, nucleus accumbens, and amygdala) were not significantly associated with cocaine use at 3-month follow-up. In addition, we found that when controlling for self-reported craving, individual differences in right dACC activity made a rather large and unique contribution to the prediction of recent cocaine use. These findings suggest that a neural correlate of attentional bias in the dACC might be a useful biomarker of cocaine use after treatment. Most importantly, as early as in the first week of treatment our findings provide information on an individual's risk to use cocaine again 3 months after treatment.

The dorsal part of the ACC has been implicated in cognitive processes such as salience detection (Seeley et al, 2007), conflict monitoring in general (Botvinick et al, 2004; Kerns et al, 2004; Egner et al, 2008) and in the presence of emotionally salient distractors (specifically the right dACC; Haas et al, 2006). Hyperactivity in the ACC associated with attentional bias to substance-related cues has previously been shown in smokers (Luijten et al, 2011, 2012; Nestor

Table 3c Bootstrap Results for Individual Predictors in the Final Model Predicting Recent Cocaine Use

\begin{tabular}{lccc}
\hline $\begin{array}{l}\text { Predictor } \\
\text { variables }\end{array}$ & $\begin{array}{c}\text { Unstandardized } \\
\text { coefficients }(=\boldsymbol{\beta})\end{array}$ & $\begin{array}{c}\text { Standard } \\
\text { error } \\
(=\mathbf{S E})\end{array}$ & $\begin{array}{c}\text { Bootstrapped 95\% } \\
\text { confidence interval }\end{array}$ \\
\hline $\begin{array}{l}\text { Craving in the week } \\
\text { before treatment } \\
\text { r-dACC }\end{array}$ & 3.82 & 1.48 & $1.48-7.36^{\mathrm{a}}$ \\
& 15.62 & 5.35 & $4.61-25.14^{\mathrm{a}}$
\end{tabular}

Abbreviations: $r$, right; dACC, dorsal anterior cingulate cortex.

aSignificant (value 0 is not in the confidence interval).

${ }^{\mathrm{b}}$ Attentional bias contrast (cocaine minus neutral).

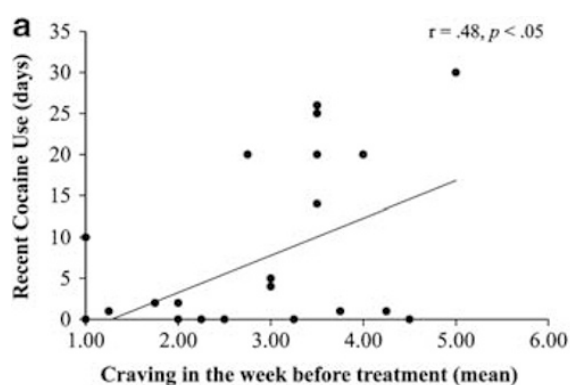

et al, 2011; Janes et al, 2010b) and alcohol-dependent patients (Vollstädt-Klein et al, 2012), and is thought to reflect enhanced conflict when task performance is being interfered by the automatic detection of salient substancerelated stimuli. However, the present design does not rule out alternative interpretations of the dACC involvement. For example, it might be possible that dACC activity is related to the greater requirement for cognitive control during the drug trials, rather than the content of the stimuli per se.

In relation to relapse, it is suggested that individual variations in the brain's ability to control cognitive conflict may account for differences in risk of relapse (Garavan and Hester, 2007). Our results support this idea by finding that when more top-down control is necessary (ie, increased dACC activity) to focus on a cognitive task in the presence of distracting drug cues one is more vulnerable to use cocaine after treatment. Additionally, individuals who need less top-down control (as reflected by less to no attentional bias-related dACC activity) are less susceptible to return to daily use of cocaine. This is in line with findings of Janes et al (2010b) who found that attentional bias-related dACC activity was predictive of relapse in smokers.

Concerning the self-report measures we examined in the current study, we found that self-reported craving in the week before treatment-but not substance use severitywas predictive of cocaine use at 3-month follow-up. Craving constitutes a central role in established theories of addiction (Robinson and Berridge, 1993). Indeed several studies have reported that subjective craving is associated with relapse and treatment outcome in cocaine-dependent patients (Weiss et al, 2003; Paliwal et al, 2008; Preston et al, 2009). More recent models of addiction propose a role for both craving and attentional bias in the maintenance of substance use and relapse (Franken, 2003; Field and Cox, 2008). Our results support this by finding that both selfreported craving in the week before treatment and individual variability in AACC activity related to attentional bias were associated with cocaine use at follow-up, suggesting a role for both processes in relapse. However, these models suggest that attentional bias predicts relapse via craving and not directly, whereas our results showed that dACC activity was a strong predictor, even when controlling for self-reported craving. This suggests that enhanced attentional bias-related brain activity might be independently associated with drug relapse.

Somewhat unexpected in the light of the recent discussion on the relation between craving and attentional bias (Field,

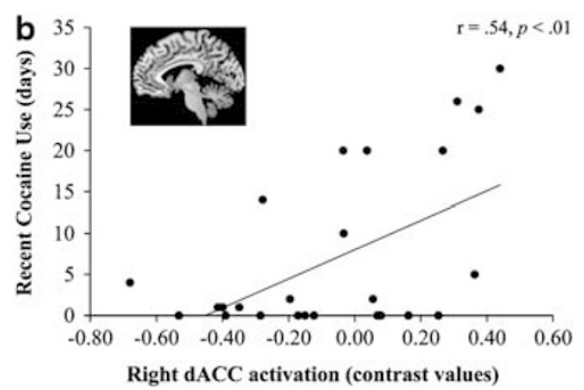

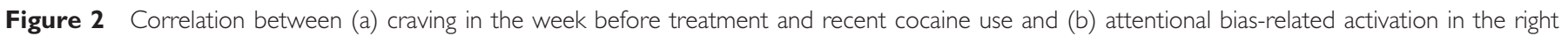
$\mathrm{dACC}$ and recent cocaine use. $\mathrm{dACC}$, dorsal anterior cingulate cortex. 
Munafo and Franken, 2009), no significant correlations between craving, attentional bias, and dACC activation were observed (see Supplementary Information, Part II for results). The meta-analysis by Field et al (2009) showed that subjective craving is consistently (though weakly) correlated with behavioral and EEG indices of attentional bias. Concerning the behavioral measures, it is possible that the statistical power of the present study was too low to yield significant results given the fact that the correlations between craving and the Stroop task are known to be weak $(r=0.15)$. In addition, dACC activation was not found to be associated with craving nor behavioral attentional bias. This is somewhat unexpected since all measures could be regarded as measures of the same underlying processing: 'motivational salience'. Although exact interpretation is not straightforward (also given the relative small $n$ ), it seems at least to be an indication that the ACC does not necessarily be the neurobiological structure responsible for cocaine craving. Further, it should be kept in mind that craving was not measured during fMRI scanning but was an average measure (OCDUS) of the craving in the week before the fMRI measurement.

The present study indicates that cocaine use after treatment is better predicted by neuroimaging than behavioral measures of the addiction Stroop effect. Other fMRI studies examining neurocognitive predictors of substance relapse have reported similar results in methamphetamine relapse (Paulus et al, 2005) and cocaine relapse (Brewer et al, 2008; Jia et al, 2011). Although previous studies have reported an association between attentional bias on the behavioral level and drug relapse (Marissen et al, 2006; Carpenter et al, 2006) we could not find any significant associations between behavioral measures and cocaine use outcome (see Supplementary Information, Part III for results). It might be that functional neuroimaging is a better predictor because it is a more sensitive measure of individual differences in biased attention to drug cues than behavioral measures.

Possible clinical implications of our results include the use of dopaminergic manipulation to reduce attentional bias-related brain activity (Ersche et al, 2010; Goldstein et al, 2010; Luijten et al, 2012) or attentional retraining (Schoenmakers et al, 2010). Patients who are vulnerable to relapse due to enhanced attentional bias for drug cues might benefit from such approaches, although these interventions need further investigation. Future intervention studies using either psychopharmacological interventions or cognitive training may obtain valuable information by identifying those patients with higher levels of dACC activity in response to drug cues.

The present study had limitations. First, we only measured days of cocaine use in the last month of the 3month follow-up period. We could not rule out that patients who reported to have used 0 days in the last month did not use in the 2 months before follow-up and could therefore not term them as 'non-relapsers'. Thus, with the present design it was not possible to predict relapse (yes $v s$ no) which makes it difficult to compare the present results with other fMRI studies on relapse prediction (eg, Janes et al, 2010b; Paulus et al, 2005). However, it has been found that continuous and dichotomous drug use outcome measures are strongly related to each other (McKay et al, 2001), suggesting that the present outcome measure does provide some information on relapse risk. To examine associations between study variables and different drug use outcome measures, future studies should use multiple outcome measures (eg, time to relapse, relapse vs non-relapse; see for a discussion also Miller et al, 1996). Second, while we found an association between enhanced attentional biasrelated brain activity and cocaine use outcome in patients after treatment, we could not demonstrate an overall withingroup attentional bias effect in the ROIs. The absence of an overall effect on the attentional bias contrast (cocaine minus neutral words) is not an unusual finding in cocaine Stroop fMRI research (Goldstein et al, 2007, 2009, 2010; Moeller et al, 2012) and may be due to the presence of individual differences in brain activity related to attentional bias (Hester and Garavan, 2009), as manifested by the fact that brain activity was predictive of relapse in a population of variable relapse risk. We would like to emphasize, however, that the task did activate regions that are typically expected to be activated for a Stroop task such as the dACC and the DLPFC for the neutral and cocaine condition separately (see Supplementary Figure 1 in Supplementary Information, Part VI). Third, we did not assess other psychiatric disorders using a structured interview or questionnaire and therefore could not examine the influence of these disorders on cocaine use outcome. Finally, we had a large number of predictors and a relatively small sample which could have led to over-fitting of the prediction model. Therefore, we performed a stepwise regression analysis to remove all redundant predictors. To reduce the probability of type II errors, we included variables in a hierarchical manner based upon previous literature (self-report measures first, behavior measures second, and neuroimaging measures third) so that the predictors are evaluated in each step separately.

In conclusion, the current study showed the clinical relevance of measuring attentional bias-related activity in the dACC in cocaine-dependent patients during the starting phase of treatment. Not only does this provide valuable information about the neurocognitive mechanisms of addiction and of addiction treatment processes, these measures may also help us identifying individuals who are at risk of relapse into cocaine use after detoxification treatment. Although direct clinical implications are not very easy to implement using fMRI methodology, ultimately findings like these may help to develop clinical profiles based on neurocognitive measures to design tailor-made follow-up treatment schedules. Future treatment intervention studies should investigate how this information might benefit relapse-vulnerable patients.

\section{ACKNOWLEDGEMENTS}

We thank Radha Jagroep, Yavuz Kilic, and Alexandra de Raaij for their assistance with data collection. This study was supported by a grant of the Netherlands Organization for Health Research and Development (ZonMw Grant no. 31160203 to IHA Franken). The funding organization had no role in study design, data collection and analysis, decision to publish, or preparation of the manuscript. 


\section{DISCLOSURE}

The authors declare no conflict of interest.

\section{REFERENCES}

Botvinick MM, Cohen JD, Carter CS (2004). Conflict monitoring and anterior cingulate cortex: an update. Trends Cogn Sci (Regul Ed) 8: 539-546.

Brewer JA, Worhunsky PD, Carroll KM, Rounsaville BJ, Potenza MN (2008). Pretreatment brain activation during stroop task is associated with outcomes in cocaine-dependent patients. Biol Psychiatry 64: 998-1004.

Carpenter KM, Schreiber E, Church S, McDowell D (2006). Drug Stroop performance: relationships with primary substance of use and treatment outcome in a drug-dependent outpatient sample. Addict Behav 31: 174-181.

Cox WM, Fadardi JS, Pothos EM (2006). The addiction-stroop test: theoretical considerations and procedural recommendations. Psychol Bull 132: 443-476.

Cox WM, Hogan LM, Kristian MR, Race JH (2002). Alcohol attentional bias as a predictor of alcohol abusers' treatment outcome. Drug Alcohol Depend 68: 237-243.

Cox WM, Pothos EM, Hosier SG (2007). Cognitive-motivational predictors of excessive drinkers' success in changing. Psychopharmacology (Berl) 192: 499-510.

Donovan DM (1996). Assessment issues and domains in the prediction of relapse. Addiction 91: S29-S36.

Efron B, Tibshirani RJ (1993). An Introduction to the Bootstrap. Chapman \& Hall: New York, NY.

Egner T, Etkin A, Gale S, Hirsch J (2008). Dissociable neural systems resolve conflict from emotional versus nonemotional distracters. Cereb Cortex 18: 1475-1484.

Ersche KD, Bullmore ET, Craig KJ, Shabbir SS, Abbott S, Müller U et al (2010). Influence of compulsivity of drug abuse on dopaminergic modulation of attentional bias in stimulant dependence. Arch Gen Psychiatry 67: 632-644.

Field A (2009). Discovering Statistics Using SPSS. 3rd edn. Sage: London, UK.

Field M, Cox WM (2008). Attentional bias in addictive behaviors: a review of its development, causes, and consequences. Drug Alcohol Depend 97: 1-20.

Field M, Munafo MR, Franken IHA (2009). A meta-analytic investigation of the relationship between attentional bias and subjective craving in substance abuse. Psychol Bull 135: 589-607.

Franken IHA (2003). Drug craving and addiction: integrating psychological and neuropsychopharmacological approaches. Prog Neuropsychopharmacol Biol Psychiatry 27: 563-579.

Franken IHA, Hendriks VM (1999). Predicting outcome of inpatient detoxification of substance abusers. Psychiatr Serv 50: 813-817.

Franken IHA, Hendriks VM, van den Brink W (2002). Initial validation of two opiate craving questionnaires-the obsessive compulsive drug use scale and the desires for drug questionnaire. Addict Behav 27: 675-685.

Friston KJ, Holmes A, Poline JB, Price CJ, Frith CD (1996). Detecting activations in PET and fMRI: levels of inference and power. Neuroimage 4: 223-235.

Garavan H, Hester R (2007). The role of cognitive control in cocaine dependence. Neuropsychol Rev 17: 337-345.

Goldstein RZ, Alia-Klein N, Tomasi D, Carrillo JH, Maloney T, Woicik PA et al (2009). Anterior cingulate cortex hypoactivations to an emotionally salient task in cocaine addiction. Proc Natl Acad Sci USA 106: 9453-9458.

Goldstein RZ, Tomasi D, Rajaram S, Cottone LA, Zhang L, Maloney $\mathrm{T}$ et al (2007). Role of the anterior cingulate and medial orbitofrontal cortex in processing drug cues in cocaine addiction. Neuroscience 144: 1153-1159.
Goldstein RZ, Volkow ND (2011). Dysfunction of the prefrontal cortex in addiction: neuroimaging findings and clinical implications. Nat Rev Neurosci 12: 652-669.

Goldstein RZ, Woicik PA, Maloney T, Tomasi D, Alia-Klein N, Shan J et al (2010). Oral methylphenidate normalizes cingulate activity in cocaine addiction during a salient cognitive task. Proc Natl Acad Sci USA 107: 16667-16672.

Haas BW, Omura K, Constable RT, Canli T (2006). Interference produced by emotional conflict associated with anterior cingulate activation. Cogn Affect Behav Neurosci 6: 152-156.

Hättenschwiler J, Ruesch P, Hell D (2000). Effectiveness of inpatient drug detoxification: links between process and outcome variables. Eur Addict Res 6: 123-131.

Hendriks VM, Kaplan CD, Van Limbeek J, Geerlings P (1989). The addiction severity index-reliability and validity in a dutch addict Population. J Subst Abuse Treat 6: 133-141.

Hester R, Garavan H (2009). Neural mechanisms underlying drugrelated cue distraction in active cocaine users. Pharmacol Biochem Behav 93: 270-277.

Janes AC, Pizzagalli DA, Richardt S, Frederick Bde B, Holmes AJ, Sousa $J$ et al (2010a). Neural substrates of attentional bias for smoking-related cues: an fMRI study. Neuropsychopharmacology 35: 2339-2345.

Janes AC, Pizzagalli DA, Richardt S, Bd Frederick, Chuzi S, Pachas $\mathrm{G}$ et al (2010b). Brain reactivity to smoking cues prior to smoking cessation predicts ability to maintain tobacco abstinence. Biol Psychiatry 67: 722-729.

Jia Z, Worhunsky PD, Carroll KM, Rounsaville BJ, Stevens MC, Pearlson GD et al (2011). An initial study of neural responses to monetary incentives as related to treatment outcome in cocaine dependence. Biol Psychiatry 70: 553-560.

Kerns JG, Cohen JD, MacDonald AW, Cho RY, Stenger VA, Carter CS (2004). Anterior cingulate conflict monitoring and adjustments in control. Science 303: 1023-1026.

Knutson B, Wimmer GE, Rick S, Hollon NG, Prelec D, Loewenstein G (2008). Neural antecedents of the endowment effect. Neuron 58: $814-822$.

Kosten TR, Scanley BE, Tucker KA, Oliveto A, Prince C, Sinha R et al (2006). Cue-induced brain activity changes and relapse in cocaine-dependent patients. Neuropsychopharmacology 31: 644-650.

Leshner AI (1997). Addiction is a brain disease, and it matters. Science 278: 45-47.

Luijten M, Veltman DJ, Hester R, Smits M, Pepplinkhuizen L, Franken IHA (2012). Brain activation associated with attentional bias in smokers is modulated by a dopamine antagonist. Neuropsychopharmacology 37: 2772-2779.

Luijten M, Veltman DJ, van den Brink W, Hester R, Field M, Smits $M$ et al (2011). Neurobiological substrate of smoking-related attentional bias. Neuroimage 54: 2374-2381.

Marhe R, Waters AJ, Van de Wetering BJM, Franken IHA (2012). Implicit and explicit drug-related cognitions during detoxification treatment are associated with drug relapse: an ecological momentary assessment study. J Consult Clin Psychol (in press).

Marissen MAE, Franken IHA, Waters AJ, Blanken P, van den Brink W, Hendriks VM (2006). Attentional bias predicts heroin relapse following treatment. Addiction 101: 1306-1312.

Mars RB, Coles MGH, Grol MJ, Holroyd CB, Nieuwenhuis S, Hulstijn W et al (2005). Neural dynamics of error processing in medial frontal cortex. Neuroimage 28: 1007-1013.

McKay JR (1999). Studies of factors in relapse to alcohol, drug and nicotine use: a critical review of methodologies and findings. J Stud Alcohol 60: 566-576.

McKay JR, Alterman AI, Koppenhaver J, Mulvaney F, Bovasso G, Ward K (2001). Continuous, categorical, and time to event cocaine use outcome variables: degree of intercorrelation and sensitivity to treatment group differences. Drug Alcohol Depend 62: $19-30$. 
McLellan AT, Luborsky L, Woody GE, O’Brien CP (1980). An improved diagnostic evaluation instrument for substance abuse patients. The Addiction Severity Index. J Nerv Ment Dis 168: 26-33.

Miller WR (1996). What is a relapse? Fifty ways to leave the wagon. Addiction 91: S15-S27.

Miller WR, Westerberg VS, Harris RJ, Tonigan JS (1996). What predicts relapse? Prospective testing of antecedent models. Addiction 91: S155-S172.

Moeller SJ, Tomasi D, Woicik PA, Maloney T, Alia-Klein N, Honorio J et al (2012). Enhanced midbrain response at 6-month follow-up in cocaine addiction, association with reduced drugrelated choice. Addict Biol 17: 1013-1025.

Mooney CZ, Duval RD (1993). Bootstrapping: A Nonparametric Approach to Statistical Inference. Sage: Newbury Park, CA.

Nestor L, McCabe E, Jones J, Clancy L, Garavan H (2011). Differences in 'bottom-up' and 'top-down' neural activity in current and former cigarette smokers: evidence for neural substrates which may promote nicotine abstinence through increased cognitive control. Neuroimage 56: 2258-2275.

Paliwal P, Hyman SM, Sinha R (2008). Craving predicts time to cocaine relapse: further validation of the Now and Brief versions of the cocaine craving questionnaire. Drug Alcohol Depend 93: 252-259.

Paulus MP, Tapert SF, Schuckit MA (2005). Neural activation patterns of methamphetamine-dependent subjects during decision making predict relapse. Arch Gen Psychiatry 62: 761-768.

Poling J, Kosten TR, Sofuoglu M (2007). Treatment outcome predictors for cocaine dependence. Am J Drug Alcohol Abuse 33: 191-206.

Powell J, Dawkins L, West R, Powell J, Pickering A (2010). Relapse to smoking during unaided cessation: clinical, cognitive and motivational predictors. Psychopharmacology (Berl) 212: 537-549.

Preston KL, Vahabzadeh M, Schmittner J, Lin J-, Gorelick DA, Epstein DH (2009). Cocaine craving and use during daily life. Psychopharmacology (Berl) 207: 291-301.
Robinson TE, Berridge KC (1993). The neural basis of drug craving: an incentive-sensitization theory of addiction. Brain Res Rev 18: 247-291.

Schoenmakers TM, de Bruin M, Lux IF, Goertz AG, Van Kerkhof DH, Wiers RW (2010). Clinical effectiveness of attentional bias modification training in abstinent alcoholic patients. Drug Alcohol Depend 109: 30-36.

Seeley WW, Menon V, Schatzberg AF, Keller J, Glover GH, Kenna $\mathrm{H}$ et al (2007). Dissociable intrinsic connectivity networks for salience processing and executive control. J Neurosci 27: 2349-2356.

Tzourio-Mazoyer N, Landeau B, Papathanassiou D, Crivello F, Etard O, Delcroix N et al (2002). Automated anatomical labeling of activations in SPM using a macroscopic anatomical parcellation of the MNI MRI single-subject brain. Neuroimage 15: 273-289.

Vollstädt-Klein S, Loeber S, Richter A, Kirsch M, Bach P, von der Goltz C et al (2012). Validating incentive salience with functional magnetic resonance imaging: association between mesolimbic cue reactivity and attentional bias in alcohol-dependent patients. Addict Biol 17: 807-816.

Waters AJ, Marhe R, Franken IHA (2012). Attentional bias to drug cues is elevated before and during temptations to use heroin and cocaine. Psychopharmacology (Berl) 219: 909-921.

Waters AJ, Shiffman S, Sayette MA, Paty JA, Gwaltney CJ, Balabanis MH (2003). Attentional bias predicts outcome in smoking cessation. Health Psychol 22: 378-387.

Weiss RD, Griffin ML, Mazurick C, Berkman B, Gastfriend DR, Frank A et al (2003). The relationship between cocaine craving, psychosocial treatment, and subsequent cocaine use. $A m \mathrm{~J}$ Psychiatry 160: 1320-1325.

Worsley KJ, Marrett S, Neelin P, Vandal AC, Friston KJ, Evans AC (1996). A unified statistical approach for determining significant signals in images of cerebral activation. Hum Brain Mapp 4: 58-73.

Supplementary Information accompanies the paper on the Neuropsychopharmacology website (http://www.nature.com/npp) 